\title{
Development of additional pituitary hormone deficiencies in pediatric patients originally diagnosed with idiopathic isolated GH deficiency
}

\author{
Werner F Blum, Cheri Deal ${ }^{1}$, Alan G Zimmermann², Elena P Shavrikova ${ }^{3}$, \\ Christopher J Child ${ }^{4}$, Charmian A Quigley ${ }^{2}$, Stenvert L S Drop ${ }^{5}$, Gordon B Cutler Jr ${ }^{2}$ \\ and Ron G Rosenfeld ${ }^{6}$ \\ Lilly Deutschland GmbH, Werner-Reimers-Strasse 2-4, 61352 Bad Homburg, Germany, 'University of Montreal and \\ CHU Ste-Justine, Montreal, Quebec, Canada, ${ }^{2}$ Lilly Research Laboratories, Indianapolis, Indiana 46285, USA, ${ }^{3} \mathrm{PSI}$ \\ Company Ltd., 191119 St Petersburg, Russia, ${ }^{4}$ Lilly Research Laboratories, Erl Wood Manor, Windlesham GU20 6PH, \\ UK, ${ }^{5}$ Department of Pediatrics, Erasmus Medical Centre Sophia, 3015-GJ Rotterdam, The Netherlands and \\ ${ }^{6}$ Department of Pediatrics, Oregon Health and Science University, Portland, Oregon 97239, USA
}

\author{
Correspondence \\ should be addressed to \\ WF Blum \\ Email \\ wernblum@gmail.com
}

\begin{abstract}
Objective: We assessed the characteristics of children initially diagnosed with idiopathic isolated GH deficiency (IGHD) who later developed additional (multiple) pituitary hormone deficiencies (MPHD).

Design: Data were analyzed for 5805 pediatric patients with idiopathic IGHD, who were GH-naïve at baseline and GH-treated in the multinational, observational Genetics and Neuroendocrinology of Short Stature International Study.

Methods: Development of MPHD was assessed from investigator diagnoses, adverse events, and concomitant medications. Analyses were performed for all patients and for those who developed MPHD within 4.5 years or had $\geq 3.5$ years, follow-up and continued to have IGHD (4-year cohort).

Results: MPHD developed in 118/5805 (2.0\%) children overall, and in 96/1757 (5.5\%) in the 4-year cohort. Patients who developed MPHD had more profound GHD, with decreased height SDS, IGF1 SDS and peak stimulated GH, and greater height decrement vs target, compared with children who continued to have IGHD $(P<0.001$ for each variable). Delivery complications, congenital anomalies, and perinatal/neonatal adverse events occurred more frequently in patients who developed MPHD. The most frequent additional deficiency was TSH (82 patients overall); four patients developed two pituitary hormone deficiencies and one developed three deficiencies. Multivariable logistic regression indicated that years of follow-up (odds ratio 1.55), baseline age (1.17), baseline height SDS (0.69), and peak stimulated GH (0.64) were associated with the development of MPHD.

Conclusions: MPHD is more likely to develop in patients with more severe idiopathic IGHD. Older baseline age, lower baseline height SDS, and longer follow-up duration are associated with increased risk of development of MPHD.

\section{Introduction}

Growth failure, with height significantly below normal for age and gender, is the predominant reason for referring children to a pediatric endocrinologist. To identify those patients who have growth hormone deficiency (GHD), appropriate endocrine tests are performed. Causes of GHD include congenital lack of somatotrophs, due to pituitary aplasia or hypoplasia, or loss/destruction of somatotrophs resulting from hypothalamic-pituitary trauma, tumor, surgery, or radiation. Children with hypopituitarism, with multiple pituitary hormone deficiencies (MPHD), have generally been found to have structural hypothalamic/pituitary abnormalities evident on magnetic
(C) 2014 European Society of Endocrinology Printed in Great Britain
Published by Bioscientifica Ltd. 
resonance imaging $(\mathrm{MRI})(1,2,3)$ or identifiable genetic causes $(3,4,5,6,7)$. However, many childhood cases of GHD are still diagnosed as idiopathic, where the true cause remains unknown, especially if the diagnostic workup does not extend beyond functional testing of $\mathrm{GH}$ secretion.

A large proportion of childhood idiopathic GHD cases continue to have isolated GHD (IGHD) as their sole manifestation of hypothalamic-pituitary dysfunction, regardless of etiology. However, some will later develop other pituitary deficiencies (8), and patients with such deficiencies may have serious or even fatal outcomes (9). It is important, therefore, to know what proportion of patients will subsequently develop MPHD, what specific deficiencies and combination of deficiencies will develop, the likely time course of the additional deficiencies, and which factors are associated with an increased risk of developing MPHD.

The multinational Genetics and Neuroendocrinology of Short Stature International Study (GeNeSIS) was designed to collect data on patients with short stature of various etiologies. From this large database, information on children initially diagnosed by the prescribing endocrinologist as having idiopathic IGHD was examined to gain insight into characteristics of patients who were subsequently diagnosed with MPHD. In addition, we examined the time course and possible predictive factors for patients who progressed to MPHD while being followed in GeNeSIS. Such evaluations may guide physicians monitoring children with idiopathic IGHD in order to implement appropriate testing and therapy.

\section{Subjects and methods}

\section{Patients}

Patient data were obtained from GeNeSIS, a prospective, open-label, multinational, observational research program (ClinicalTrials.gov, NCT01088412). The study collects information on clinical management and treatment outcomes of pediatric patients with growth disorders, as documented by the clinician during routine endocrine practice. Patients who are receiving or who will start GH (Humatrope; Eli Lilly and Company) may enroll in GeNeSIS and investigators are encouraged to continue patients in the study regardless of changes in brand or discontinuation of $\mathrm{GH}$. Institutional review board approval was obtained and all applicable regulatory requirements in the participating countries were followed for this post-marketing study, which is conducted

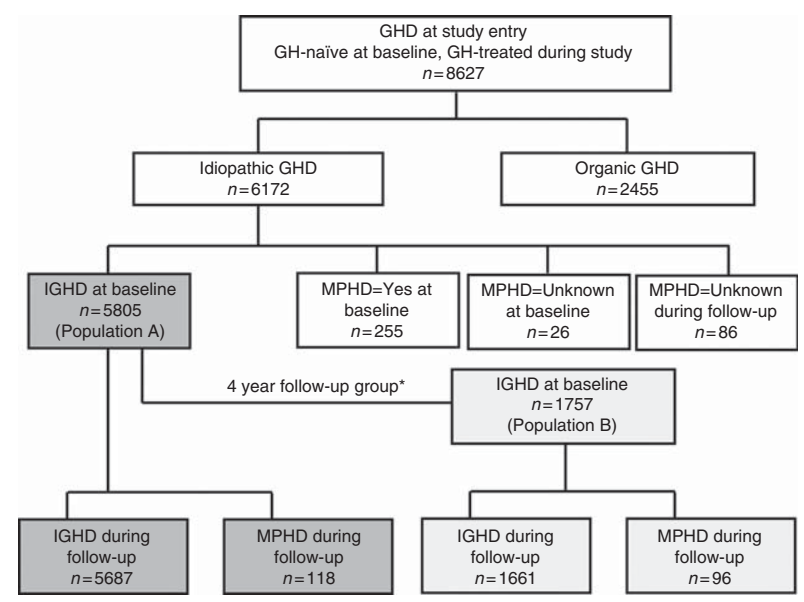

Figure 1

Study disposition showing numbers of pediatric patients identified as having idiopathic isolated GH deficiency (IGHD) and who developed multiple pituitary hormone deficiencies (MPHD) during follow-up under GH treatment. *Population A included all patients who were considered to have IGHD at entry to the study; Population B included patients considered to have IGHD at entry and who either developed MPHD within 4.5 years of follow-up or continued to have IGHD and had at least 3.5 years of follow-up in the study.

according to the ethical principles of the Declaration of Helsinki. Written consent for data collection, electronic processing, and publication were obtained from parents or guardians of enrolled patients, in accordance with national requirements.

The growth disorder of patients in GeNeSIS may be due to a number of etiologies, but for the purpose of this analysis patients were only included if they had idiopathic GHD and were GH treatment-naïve at study entry. GeNeSIS is an observational study, with no specific tests or procedures required. Thus, all information, including the diagnosis of GHD, deficiency of other pituitary hormones, and requirements for MRI or other tests, were as reported by the investigator. Patients with a non-GHD cause of growth failure or with GHD due to an organic cause were excluded from the analysis. Organic GHD included congenital causes such as abnormal pituitary-hypothalamic development (diagnosed by MRI) or genetic defects, and GHD acquired as a result of tumor, radiation, or surgery (Fig. 1). As shown in Supplementary Table 1 , see section on supplementary data given at the end of this article, the countries with the greatest numbers of patients with idiopathic IGHD enrolled were the USA (1935 patients, 31.4\%), Germany 
(1055, 17.1\%), and Japan (1039, 16.8\%). Patients were evaluated at baseline and at $\sim 6$-month intervals according to the discretion of the investigator.

\section{Methods}

All data were collected on study case report forms. At baseline, investigators were asked to indicate the pituitary hormone status for adrenocorticotrophic hormone (ACTH), anti-diuretic hormone (ADH), luteinizing hormone/follicle-stimulating hormone (LH/FSH), prolactin (PRL), and thyroid-stimulating hormone (TSH), as abnormally low, normal, or abnormally high. At each study visit, investigators were asked to document the occurrence of primary or secondary hypothyroidism and other adverse events, pubertal development, and treatment with concomitant medications, using specific case report forms. Conditions present before GH therapy were classified as pre-existing, whilst those that occurred or worsened after starting GH therapy were classified as adverse events. Adverse events were categorized according to the Medical Dictionary for Regulatory Activities (MedDRA, version 11.0).

The information on pituitary hormone status was collected on four levels: i) direct assignment by case report form check-box at baseline; ii) direct assignment by checkbox at any visit (primary or secondary hypothyroidism, spontaneous or induced puberty); iii) pre-existing medical condition/adverse event term indicating a pituitary hormone deficiency (e.g., ACTH deficiency); and iv) concomitant medication term indicating presumptive pituitary hormone deficiency (e.g. thyroxine or cortisol), which were reviewed on an individual patient basis. If information was inconsistent, direct information (check-box) was given priority over pre-existing medical condition/adverse event terms, which were given priority over concomitant medication terms indicating hormone replacement therapy. The rationale for this hierarchy was based on the presumptive reliability of the three methods of data collection in the case report forms.

Assignment of pituitary hormone deficiency based upon concomitant medications was performed as follows. Thyroid hormone replacement at study baseline, without a pre-existing diagnosis or clear diagnostic evidence of primary hypothyroidism, was classified as TSH deficiency, and the patient was thus excluded from analysis as having baseline MPHD. If thyroid hormone replacement began after starting GH therapy, and there was no pre-existing diagnosis of secondary hypothyroidism or other evidence of TSH deficiency, the TSH status was assigned as unknown to avoid misclassifying thyroxine treatment for primary hypothyroidism as an incident TSH deficiency. Similarly, cortisol replacement at baseline was counted as ACTH deficiency, and the patient was excluded from analysis; during GH therapy, the ACTH status was assigned as unknown for incident cortisol treatment without other proof of ACTH deficiency, in order not to misclassify other possible reasons for cortisol administration as an incident ACTH deficiency (only one patient in this category). Estrogen treatment to induce puberty in girls younger than 14 years of age and testosterone treatment for more than 1 year to induce puberty in boys were considered as evidence of LH/FSH deficiency. The age criterion of 14 years in girls was introduced to be as stringent as possible for identifying LH/FSH deficiency based on estrogen treatment, because estrogen-containing medication for contraception could not be distinguished from estrogen replacement for hypogonadotrophic hypogonadism based on the available data. LH/FSH deficiency was excluded if the patient entered puberty spontaneously (Tanner stage 2 or above) or received GNRH agonist or aromatase inhibitor treatment to delay epiphyseal fusion. $\mathrm{ADH}$ deficiency was assigned if a patient began $\mathrm{ADH}$ agonist treatment during GH therapy and did not have an event term indicating enuresis.

Any deficiency of ACTH, ADH, LH/FSH, PRL, or TSH in addition to GHD was classified as MPHD. Three categories of MPHD status were defined: $\mathrm{MPHD}=$ yes (where data indicated any additional deficiency), MPHD = no (where data indicated absence of such deficiencies), and MPHD= unknown (where data were unclear).

Inclusion in this analysis required that patients had IGHD at baseline (i.e., MPHD=no) and a defined MPHD status at follow-up (MPHD=yes or no). Patients who had MPHD or ambiguous pituitary status $(\mathrm{MPHD}=$ yes or unknown) at baseline, or ambiguous pituitary status $(\mathrm{MPHD}=$ unknown) at follow-up, were excluded from this analysis.

\section{Statistical analysis}

It was expected that observations of additional pituitary hormone deficiencies would increase with time on study. Therefore, to compare features of patients who did or did not develop MPHD over a similar period of observation, a subgroup of patients (Population B; 4-year follow-up cohort) was defined as those who developed MPHD within 4.5 years from baseline or were followed for $\geq 3.5$ years without developing MPHD. 
When more than one stimulation test was used for the diagnosis of GHD, the higher peak GH value was retained for analysis; $\mathrm{GH}$ concentrations reported as $\mathrm{mU} / \mathrm{l}$ were converted to $\mu \mathrm{g} / \mathrm{l}$ by dividing by 3 . Calculation of height SDS was based on US general population data (10) for all patients, except Japanese patients when the calculation was based on Japanese standards (11). Bone age was determined by the method of Greulich \& Pyle (12) or, if determined by the Tanner-Whitehouse method, was converted to a Greulich-Pyle value as previously described (13). Bone age SDS was calculated as bone age minus chronological age divided by the s.D. for bone age from the tables of Greulich \& Pyle.

The primary comparison was between patients who continued to have IGHD and those who developed MPHD. Baseline between-group comparisons used ANOVA for continuous variables, with pituitary hormone status as the explanatory variable, and Fisher's exact test for categorical variables. Continuous variables are expressed as mean with s.D., except peak stimulated GH concentrations, which were compared after natural logarithmic transformation to achieve uniformity of variance, and are

Table 1 Characteristics of patients with isolated GH deficiency (IGHD) at baseline, split according to those who developed multiple hormone deficiency (MPHD) at any time during the study vs those who continued to have IGHD, for all patients (Population A), and for patients with $\geq 3.5$ years follow-up without developing MPHD (IGHD) or who developed MPHD in $\leq 4.5$ years (Population B). Values are mean \pm s.D. except for peak $\mathrm{GH}$, which shows median $(\mathrm{Q} 1, \mathrm{Q} 3)$; note that $\mathrm{GHRH}$ stimulation tests were excluded from the analysis of peak $\mathrm{GH}$.

\begin{tabular}{|c|c|c|c|c|c|}
\hline & \multicolumn{2}{|c|}{ MPHD } & \multicolumn{2}{|c|}{ IGHD } & \multirow[b]{2}{*}{$P$ value } \\
\hline & $n$ & Value & $n$ & Value & \\
\hline \multicolumn{6}{|l|}{ Population A } \\
\hline Total $n$ & & 118 & & 5687 & \\
\hline Gender, male/female (\%) & 118 & $64.4 / 35.6$ & 5678 & $67.0 / 33.0$ & 0.557 \\
\hline Age at diagnosis of GHD (years) & 118 & $9.6 \pm 4.1$ & 5658 & $10.0 \pm 3.5$ & 0.188 \\
\hline \multicolumn{6}{|l|}{ Variables at start of GH therapy } \\
\hline Age (years) & 118 & $10.0 \pm 4.1$ & 5680 & $10.4 \pm 3.5$ & 0.233 \\
\hline Bone age SDS & 87 & $-2.6 \pm 1.8$ & 3744 & $-2.0 \pm 1.5$ & $<0.001$ \\
\hline Height SDS & 118 & $-2.9 \pm 1.1$ & 5648 & $-2.4 \pm 0.9$ & $<0.001$ \\
\hline Height SDS - target height SDS & 109 & $-2.5 \pm 1.2$ & 5169 & $-1.8 \pm 1.1$ & $<0.001$ \\
\hline BMI SDS & 118 & $-0.6 \pm 1.9$ & 5589 & $-0.4 \pm 1.6$ & 0.195 \\
\hline Peak GH $(\mu \mathrm{g} / \mathrm{l})$ & 111 & $4.6(2.0,8.3)$ & 5415 & $7.0(4.6,9.7)$ & $<0.001 *$ \\
\hline IGF1 SDS & 42 & $-3.6 \pm 3.0$ & 1790 & $-2.2 \pm 2.1$ & $<0.001$ \\
\hline \multicolumn{6}{|l|}{ Variables during GH therapy } \\
\hline Initial GH dose (mg/kg per week) & 115 & $0.22 \pm 0.06$ & 5505 & $0.24 \pm 0.08$ & 0.003 \\
\hline First year gain in height SDS & 112 & $0.8 \pm 0.7$ & 4592 & $0.5 \pm 0.4$ & $<0.001$ \\
\hline First year height velocity ( $\mathrm{cm} /$ year) & 112 & $9.6 \pm 2.9$ & 4598 & $8.7 \pm 2.4$ & $<0.001$ \\
\hline First year height velocity SDS & 110 & $3.2 \pm 2.8$ & 4576 & $2.3 \pm 2.2$ & $<0.001$ \\
\hline Time on study (years) & 118 & $5.1 \pm 3.1$ & 5670 & $2.6 \pm 2.1$ & $<0.001$ \\
\hline \multicolumn{6}{|l|}{ Population B } \\
\hline Total $n$ & & 96 & & 1661 & \\
\hline Gender, male/female (\%) & 96 & $65.6 / 34.4$ & 1661 & $67.3 / 32.7$ & 0.733 \\
\hline Age at diagnosis of GHD (years) & 96 & $10.4 \pm 3.9$ & 1659 & $8.8 \pm 3.3$ & $<0.001$ \\
\hline \multicolumn{6}{|l|}{ Variables at start of $\mathrm{GH}$ therapy } \\
\hline Age at start of GH (years) & 96 & $10.8 \pm 43.9$ & 1661 & $9.2 \pm 3.2$ & $<0.001$ \\
\hline Bone age SDS & 69 & $-2.5 \pm 1.8$ & 1108 & $-2.3 \pm 1.4$ & 0.242 \\
\hline Height SDS & 96 & $-2.9 \pm 1.1$ & 1656 & $-2.4 \pm 0.8$ & $<0.001$ \\
\hline Height SDS - target height SDS & 89 & $-2.4 \pm 1.1$ & 1552 & $-1.8 \pm 1.0$ & $<0.001$ \\
\hline BMI SDS & 96 & $-0.6 \pm 1.8$ & 1632 & $-0.7 \pm 1.4$ & 0.753 \\
\hline Peak GH $(\mu \mathrm{g} / \mathrm{l})$ & 90 & $6.2(2.3,8.9)$ & 1618 & $7.7(5.3,10.2)$ & $<0.001 *$ \\
\hline IGF1 SDS & 37 & $-3.6 \pm 3.2$ & 581 & $-2.0 \pm 2.0$ & $<0.001$ \\
\hline \multicolumn{6}{|l|}{ Variables during GH therapy } \\
\hline Initial GH dose (mg/kg per week) & 94 & $0.23 \pm 0.06$ & 1602 & $0.23 \pm 0.07$ & 0.830 \\
\hline First year gain in height SDS & 92 & $0.7 \pm 0.6$ & 1568 & $0.6 \pm 0.4$ & $<0.001$ \\
\hline First year height velocity (cm/year) & 92 & $9.5 \pm 2.9$ & 1568 & $8.7 \pm 2.1$ & $<0.001$ \\
\hline First year height velocity SDS & 90 & $3.0 \pm 2.5$ & 1566 & $2.5 \pm 2.1$ & 0.019 \\
\hline Time on study (years) & 96 & $4.2 \pm 2.4$ & 5670 & $5.2 \pm 1.7$ & $<0.001$ \\
\hline
\end{tabular}


presented as median with first and third quartiles (Q1 and Q3). For patients who developed MPHD during the study, the time course for specific hormone deficiencies was analyzed by the Kaplan-Meier method. To identify predictors of developing MPHD, logistic multiple regression analysis was performed, with varied sets of explanatory variables; odds ratio (OR) and 95\% CI are presented. Two-sided $P$ values $<0.05$ were considered statistically significant. All analyses were performed using SAS procedures (version 9.1; SAS Institute, Inc., Cary, NC, USA).

\section{Results}

\section{Characteristics of analysis populations}

Initially, 6172 patients (67\% males and 33\% females) with a diagnosis of idiopathic GHD, who were GH-treatment naïve at study entry and who received GH treatment during the study, were reviewed for their pituitary hormone status (Fig. 1). Among these 6172 patients, 255 (4.1\%) were determined to have MPHD at baseline; MPHD status was unknown for $26(0.4 \%)$ patients at baseline and $86(1.4 \%)$ during follow-up. MPHD diagnosis at baseline was determined, in prespecified order of priority, from the hormone status check-box in 219 of 255 (85.9\%) patients, pre-existing condition/adverse event term in 13 (5.1\%) patients, and documented hormone replacement therapy in 23 (9.0\%) patients. Assignment of MPHD during followup was based on information from the check-boxes in $69.5 \%$ of patients, adverse event term in $13.6 \%$, and hormone replacement medication in $16.9 \%$.

The final study population, with IGHD at baseline and known MPHD status at follow-up, comprised 5805 patients. Idiopathic GHD was reported as 'classic' (low GH response to stimulation test) for $90.7 \%$ and as neurosecretory dysfunction for 9.3\%. Of the 5805 patients, 118 (2.0\%) developed MPHD during follow-up, while $5687(98.0 \%)$ continued to have IGHD (Table 1, Population A). Of the 118 patients who developed MPHD, ten were reported to have GHD due to neurosecretory dysfunction. Age at diagnosis and at start of GH therapy did not differ between patients who developed MPHD and those who continued to have IGHD. Patients who developed MPHD were significantly shorter (height SDS $P<0.001)$ and had significantly greater height decrement in relation to target height $(P<0.001)$ at the start of $\mathrm{GH}$. IGF1 SDS was significantly lower in patients who developed MPHD and peak GH in stimulation tests was also significantly lower $(P<0.001$ for comparison of
Ln-transformed values). Twenty of the 118 patients who developed MPHD had stimulated peak GH $>10 \mu \mathrm{g} / \mathrm{l}$. Patients who developed MPHD had significantly greater first-year response to GH therapy (height velocity and height SDS gain, $P<0.001)$. Gestational duration did not differ (MPHD 39.0 \pm 1.9 weeks and IGHD 38.8 \pm 2.5 weeks, $P=0.341$ ), and gestation and birth parameters were similar. Breech presentation and adverse events at the time of birth were reported significantly more frequently for patients who developed MPHD than those who continued to have IGHD (Table 2, Population A).

Mean ( \pm s.D.) duration of follow-up was longer for the group who developed MPHD (5.1 \pm 3.1 years) compared with those who continued to have IGHD $(2.6 \pm 2.1$; $P<0.001)$. Therefore, to compare features of patients who did or did not develop MPHD over a similar period of observation, a subgroup of patients (Population B; 4-year follow-up cohort, Fig. 1) was defined who developed MPHD within 4.5 years from baseline or were followed for $\geq 3.5$ years without developing MPHD. Population B comprised 1757 patients (67\% males and 33\% females), and when compared with Population A, were slightly younger at diagnosis and GH initiation (Supplementary Table 2, see section on supplementary data given at the end of this article), but other baseline characteristics were similar.

The 4-year follow-up cohort included 96 (5.5\%) patients who developed MPHD and 1661 who continued to have IGHD. Average follow-up was $4.2 \pm 2.4$ years for the patients who developed MPHD and $5.2 \pm 1.7$ years for

Table 2 Perinatal and neonatal history of complications in patients with isolated GH deficiency (IGHD) vs those who developed multiple pituitary hormone deficiencies (MPHD) at any time during the study (Population A), and in those who had $\geq 3$.5 years follow-up and continued to have IGHD vs those who developed MPHD in $\leq 4.5$ years (Population B).

\begin{tabular}{|c|c|c|c|}
\hline & $\begin{array}{l}\text { MPHD } \\
(n(\%))\end{array}$ & $\begin{array}{l}\text { IGHD } \\
(n(\%))\end{array}$ & $P$ value \\
\hline \multicolumn{4}{|l|}{ Population A } \\
\hline Total $n$ & 118 & 5687 & \\
\hline Congenital anomaly & $6(5.1)$ & $165(2.9)$ & 0.100 \\
\hline Breech presentation & $15(12.7)$ & $296(5.2)$ & $<0.001$ \\
\hline Delivery complications & $8(6.8)$ & $212(3.7)$ & 0.095 \\
\hline Perinatal asphyxia & $10(8.5)$ & $115(2.0)$ & $<0.001$ \\
\hline Neonatal complications & $18(15.3)$ & $328(5.8)$ & $<0.001$ \\
\hline \multicolumn{4}{|l|}{ Population B } \\
\hline Total $n$ & 96 & 1661 & \\
\hline Congenital anomaly & $5(5.2)$ & $57(3.4)$ & 0.335 \\
\hline Breech presentation & $12(12.5)$ & $62(3.7)$ & $<0.001$ \\
\hline Delivery complications & $6(6.3)$ & $76(4.6)$ & 0.428 \\
\hline Perinatal asphyxia & $9(9.4)$ & 32 (1.9) & $<0.001$ \\
\hline Neonatal complications & $15(15.6)$ & $110(6.6)$ & $<0.001$ \\
\hline
\end{tabular}


A

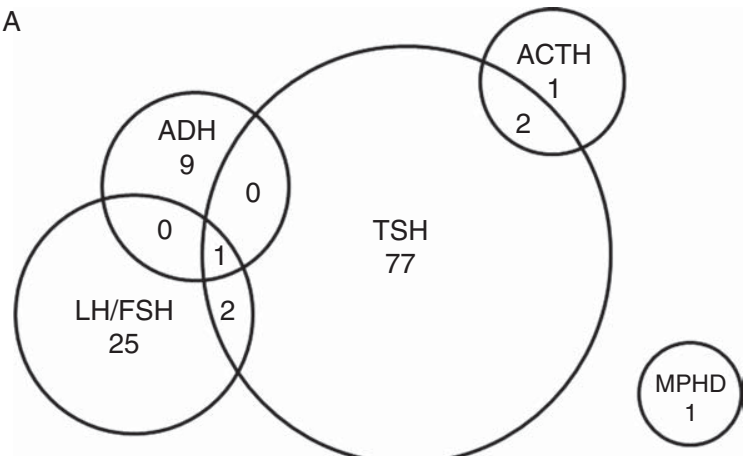

ACTH status was unknown for two patients with known TSH deficiency, the two patients with $\mathrm{LH} / \mathrm{FSH}$ plus TSH deficiencies and the patient with $\mathrm{LH} / \mathrm{FSH}$

TSH plus ADH deficiencies

TSH status was unknown for one patient with ADH deficiency and one patient with LH/FSH deficiency

One patient started thyroxine and cortisol treatment and was categorized as MPHD but no pituitary hormone deficiency was specified

$\mathrm{B}$

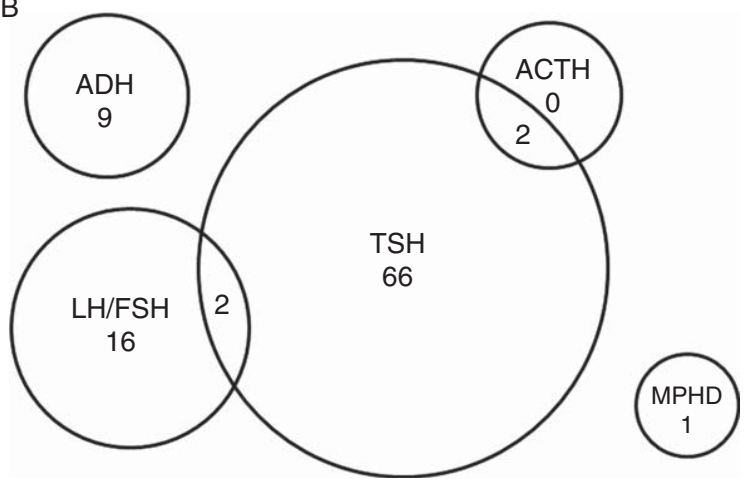

ACTH status was unknown for two patients with known TSH deficiency and the two patients with LH/FSH plus TSH deficiency

TSH status was unknown for one patient with ADH deficiency

One patient started thyroxine and cortisol treatment and was categorized as MPHD but no pituitary hormone deficiency was specified

\section{Figure 2}

Numbers of patients who entered the study with idiopathic isolated GH deficiency (IGHD) and subsequently developed the specified additional pituitary hormone deficiencies at any time in the study (A) or within 4.5 years of entry (B). All patients were GH-naïve at study entry and first started GH treatment at enrolment; patients who developed MPHD at any time were from Population A, and patients who developed MPHD within 4.5 years were from Population $B$.

those who continued to have IGHD. Age at diagnosis and age at start of GH therapy were both significantly greater in patients who developed MPHD vs those who continued to have IGHD, but bone age did not differ (Table 1, Population B). Height SDS was lower, the decrement from target height SDS was greater, and IGF1 SDS and peak stimulated GH were lower, indicating more severe GHD in the patients who developed MPHD than in those who did not. Of the 96 patients who developed MPHD, 19 had a peak stimulated $\mathrm{GH}$ concentration $>10 \mu \mathrm{g} / \mathrm{l}$. The GH-induced increase in height velocity SDS and firstyear gain in height SDS were both significantly greater in patients who developed MPHD.

In the 4-year follow-up cohort, gestational duration did not differ (MPHD 38.4 \pm 2.0 weeks and IGHD $38.9 \pm 2.3$ weeks). However, breech presentation, perinatal asphyxia, and neonatal complications all occurred significantly more frequently in the patients subsequently diagnosed with MPHD (Table 2, Population B).

\section{Additional pituitary hormone deficiencies}

Of the 118 patients who developed MPHD at any time during follow-up (Fig. 2A), 112 developed only one additional deficiency, four developed two additional deficiencies, and one patient developed three further deficiencies; for one patient only, classified as MPHD because of a reported event of hypopituitarism, and concomitant therapy with thyroxine and cortisol, no further information was available to specify which additional hormone/s were deficient. The most frequent additional pituitary hormone deficiency was TSH (82 patients, 69\%). None of the patients was reported to have PRL deficiency.

The additional pituitary hormone deficiencies that developed in the 4-year follow-up cohort (Population B) are shown in Fig. 2B. Of the 96 patients who developed MPHD, 91 (94.8\%) developed only one additional

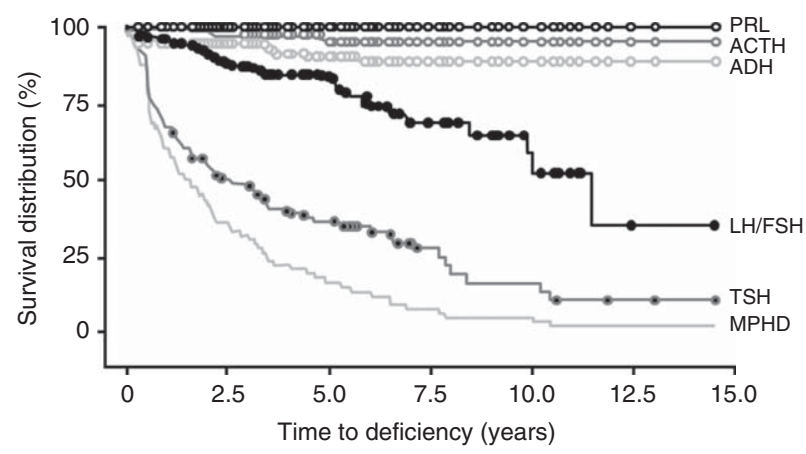

\section{Figure 3}

Kaplan-Meier plot for pediatric patients who initially had isolated GH deficiency and developed MPHD, showing time of development of specified additional pituitary hormone deficiencies and of any pituitary hormone deficiency (MPHD). Points show censored data for each individual deficiency in Population A. 
Table 3 Age at growth disorder diagnosis and onset of pituitary deficiency for patients who developed a deficiency additional to GH deficiency (MPHD) at any time in the study $(n=118)^{\mathrm{a}}$. Values shown are median $(\mathrm{Q} 1, \mathrm{Q} 3)$.

Age at diagnosis of growth disorder (years)
Age at onset of pituitary hormone
deficiency (years)
Time from GHD diagnosis to onset of
pituitary hormone deficiency (years)

$\begin{array}{r}\hline \text { TSH }(n=82) \\ \hline 9.7(5.8,12.9) \\ 12.7(9.0,14.8) \\ 1.9(0.7,4.1) \\ \hline\end{array}$

\begin{tabular}{c}
\hline LH/FSH $(n=28)$ \\
\hline $10.6(7.6,13.6)$ \\
$14.6(14.1,16.1)$ \\
$3.3(2.0,6.1)$ \\
\hline
\end{tabular}

\begin{tabular}{c}
\hline \multicolumn{1}{c}{ ADH $(n=10)$} \\
\hline $10.1(7.7,11.9)$ \\
$13.3(11.9,14.0)$
\end{tabular}

$2.4(0.5,5.2)$

\begin{tabular}{c} 
ACTH $(n=3)$ \\
\hline $5.8(5.3,6.1)$ \\
$8.0(7.8,10.9)$
\end{tabular}

$2.4(2.0,2.7)$

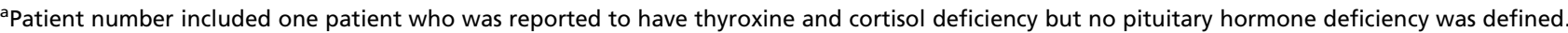

hormone deficiency and four patients developed two additional deficiencies. The additional hormone deficiency that developed most frequently was TSH deficiency (70 patients $(72.9 \%)$ ), followed by gonadotropin deficiency (18 patients (18.8\%), including two with concomitant TSH deficiency), ADH deficiency (nine patients $(9.4 \%)$ ), and ACTH deficiency (two patients (2.1\%), both with concomitant TSH deficiency).

The temporal pattern of the development of pituitary hormone deficiencies additional to GHD is shown in Fig. 3 for all patients who developed MPHD (Population A). Median age at diagnosis of each additional pituitary hormone deficiency was 8.0 years for ACTH, 12.7 years for TSH, 13.3 years for $\mathrm{ADH}$, and 14.6 years for LH/FSH (Table 3 ). Median interval from diagnosis of GH deficiency to additional pituitary deficiency was 1.9 years for TSH, 2.4 years for both ADH and ACTH, and 3.3 years for LH/FSH.

Multivariable logistic regression analysis for factors potentially predictive of development of MPHD (Supplementary Table 3 , see section on supplementary data given at the end of this article), using the 118 patients who developed MPHD and 5805 patients who continued to have IGHD (Population A), indicated the following significant factors for MPHD development: number of years of follow-up (OR: 1.55, 95\% CI 1.43-1.68); baseline age in years $(1.17,1.09-1.25)$; baseline height SDS $(0.69$, 0.58-0.83); and Ln-transformed peak stimulated GH concentration $(0.64,0.54-0.88)$. There were no statistically significant effects of gender, baseline bone age relative to chronological age, or baseline height SDS minus target height SDS. The same factors shown to be significant for the total study population were similarly significant for the 4-year follow-up cohort (Population B, data not shown).

\section{Discussion}

In this large cohort of children originally diagnosed as having idiopathic IGHD, MPHD developed in 2.0\% overall, and among children followed for a minimum of 3.5 years MPHD developed in 5.5\%. The duration of follow-up was the strongest predictor of MPHD development, and further increases in MPHD are, therefore, likely with additional follow-up time. Other predictors of MPHD were higher baseline age and more severe GHD, as reflected by lower baseline height SDS and peak stimulated GH; children who developed MPHD also had a better first-year response to GH therapy. In addition, breech presentation, perinatal asphyxia, and neonatal complications were reported significantly more frequently in patients who developed MPHD. The relative frequency of additional deficiencies that developed in these patients initially diagnosed with idiopathic IGHD was TSH greater than $\mathrm{LH} / \mathrm{FSH}$, greater than $\mathrm{ADH}$, greater than ACTH.

Our observations confirm the longstanding recommendation that indefinite follow-up for additional pituitary deficiencies is required, even among children currently diagnosed as idiopathic IGHD in routine clinical practice (14). The most frequent additional deficiency observed was TSH deficiency, which may reduce the response to GH therapy (15). Although incident hypothyroidism within the first year of GH treatment has been reported to reflect unmasking of pre-existing hypothyroidism in adult GH-deficient patients (16), the evidence in pediatric patients is more equivocal $(17,18)$; the increasing prevalence of TSH deficiency with longer follow-up in our study is more consistent with the evolution of new deficits. The second most frequent additional pituitary hormone deficiency was LH/FSH, which occurred about one-third as frequently as TSH deficiency. This is probably an underestimate because detection is most likely at the age of normal puberty and many study patients remained within the prepubertal age range.

ACTH deficiency was diagnosed during follow-up in only three patients, of whom two had concomitant TSH deficiency, consistent with previous observations $(19,20)$. However, one patient was diagnosed with ACTH deficiency as the sole additional deficiency, 
emphasizing the need to remain vigilant for such cases. The unexpected observation of ADH as the sole additional deficiency in nine patients may relate to the association of ADH deficiency and histiocytosis (21), and MRI may potentially identify the structural lesion in such cases (22).

Patients with additional pituitary deficiencies diagnosed during follow-up had a higher incidence of breech presentation, perinatal asphyxia, and neonatal complications, and a trend toward greater delivery complications. Historically, these features have been recognized as more common among children who present initially with MPHD $(23,24)$, although they are typically associated with organic rather than idiopathic GHD $(25,26)$. Thus, there appears to be a spectrum of severity among patients with such perinatal histories, with the most severe presenting initially with MPHD and the less severe presenting initially with IGHD and later progressing to MPHD.

As a group, patients diagnosed with MPHD after initiation of GH therapy had more severe GHD than those who continued to have IGHD. Importantly, lack of severe GHD does not appear to exclude future development of MPHD; ten patients who developed MPHD were initially diagnosed as having GH neurosecretory dysfunction and 20 patients had peak GH concentrations above the generally used diagnostic cut-off for GHD of $10 \mu \mathrm{g} / \mathrm{l}$ (including four of the ten diagnosed with neurosecretory dysfunction). Thus, approximately onequarter of the patients who developed MPHD lacked evidence of severe GHD at diagnosis.

While this study involved a large cohort of patients, a limitation was that it was observational and the analyzed data relied on information provided at the discretion of the investigators. The approach to define MPHD was deliberately conservative, which may have meant that a small number of patients could have had pituitary hormone deficiencies that were undetected at study entry. In addition, $4 \%$ of the patients already had MPHD at baseline and were excluded from the analyses; some of these could have had IGHD initially, but developed additional pituitary deficiencies before study entry. MRI prior to initiation of GH treatment would possibly have alerted the physician to a greater risk of future additional deficiencies. However, patients who had received an MRI and in whom an abnormal pituitary-hypothalamic morphology was found were classified as organic GHD and were excluded from this analysis. The patients with idiopathic GHD in this study either did not receive MRI or had a normal MRI finding. Therefore, this study should not be interpreted as indicating the future likelihood of MPHD among patients who have been diagnosed according to the most rigorous available methods. Rather, this study indicates the prognostic factors and frequency of subsequent MPHD in a large multinational cohort of idiopathic IGHD patients whose initial diagnosis was made according to prevailing clinical practice.

We conclude that future development of MPHD remains an important risk in patients who are diagnosed as having idiopathic IGHD. Although the current study identified both clinical and laboratory features that could predict greater risk for future MPHD development, there remained a substantial proportion of patients who developed MPHD without obvious prognostic factors. Thus, it remains important to repeatedly look for additional pituitary hormone deficiencies over an extended period for all patients. TSH was the most prevalent additional pituitary hormone deficiency and, if unrecognized, can impair the response to GH treatment. $\mathrm{ADH}$ as a sole additional deficiency also occurred and, although not reported as being associated with histiocytosis in this observational study, we emphasize that MRI can reveal structural histiocytosis lesions in such cases. Lastly, the continuing development of additional pituitary hormone deficiencies highlights the need for improved early diagnosis, which should include more thorough evaluation of pituitary function, genetics, and hypothalamic-pituitary structure by MRI.

\section{Supplementary data}

This is linked to the online version of the paper at http://dx.doi.org/10.1530/ EJE-13-0643.

\section{Declaration of interest}

W F Blum, A G Zimmermann, C J Child, and C A Quigley are employees of Eli Lilly and Company. G B Cutler is a former employee of and a consultant to Eli Lilly and Company (current address Gordon Cutler Consultancy LLC, Deltaville, VA 23043-2172, USA). C Deal, E P Shavrikova, S L S Drop, and R G Rosenfeld were members of the GeNeSIS International Advisory Board and have received honoraria and meeting expenses from Eli Lilly and Company. The study was sponsored by Eli Lilly and Company.

\section{Funding}

The Genetics and Neuroendocrinology of Short Stature International Study (GeNeSIS) is sponsored by Eli Lilly and Company.

\section{Acknowledgements}

The authors thank all investigators, patients, and their families participating in GeNeSIS. Additionally, the authors thank Dr Peter Bates, Cambridge Medical Writing Services, UK, for assistance in preparation and editorial review of the manuscript, funded by Eli Lilly and Company. 


\section{References}

1 Jagtap VS, Acharya SV, Sarathi V, Lila AR, Budyal SR, Kasaliwal R, Sankhe SS, Bandgar TR, Menon PS \& Shah NS. Ectopic posterior pituitary and stalk abnormality predicts severity and coexisting hormone deficiencies in patients with congenital growth hormone deficiency. Pituitary 201215 243-250. (doi:10.1007/s11102-011-0321-4)

2 Maghnie M, Ghirardello S \& Genovese E. Magnetic resonance imaging of the hypothalamus-pituitary unit in children suspected of hypopituitarism: who, how and when to investigate. Journal of Endocrinological Investigation 200427 496-509.

3 Mehta A, Hindmarsh PC, Mehta H, Turton JP, Russell-Eggitt I, Taylor D, Chong WK \& Dattani MT. Congenital hypopituitarism: clinical, molecular and neuroradiological correlates. Clinical Endocrinology 2009 71 376-382. (doi:10.1111/j.1365-2265.2009.03572.x)

4 Castinetti F, Reynaud R, Saveanu A, Barlier A \& Brue T. Genetic causes of combined pituitary hormone deficiencies in humans. Annales d'Endocrinologie 201273 53-55. (doi:10.1016/j.ando.2012.03.025)

5 Wit JM, Kiess W \& Mullis P. Genetic evaluation of short stature. Best Practice \& Research. Clinical Endocrinology \& Metabolism 201125 1-17. (doi:10.1016/j.beem.2010.06.007)

6 Pfaeffle R \& Klammt J. Pituitary transcription factors in the aetiology of combined pituitary hormone deficiency. Best Practice \& Research. Clinical Endocrinology \& Metabolism 201125 43-60. (doi:10.1016/j.beem.2010.10.014)

7 Pfäffle R \& Blum WF. Understanding the Genetics of Growth Hormone Deficiency: a Reference Guide. Abingdon, UK: TMG Healthcare Publications, Ltd, 2000.

8 Lange M, Feldt-Rasmussen U, Svendsen OL, Kastrup KW, Juul A \& Muller J. High risk of adrenal insufficiency in adults previously treated for idiopathic childhood onset growth hormone deficiency. Journal of Clinical Endocrinology and Metabolism $2003 \mathbf{8 8}$ 5784-5788. (doi:10.1210/jc.2003-030529)

9 Darzy KH \& Shalet SM. Hypopituitarism following radiotherapy revisited. Endocrine Development 200915 1-24.

10 Ogden CL, Kuczmarski RJ, Flegal KM, Mei Z, Guo S, Wei R, GrummerStrawn LM, Curtin LR, Roche AF \& Johnson CL. Centers for Disease Control and Prevention 2000 growth charts for the United States: improvements to the 1977 National Center for Health Statistics version. Pediatrics 2002109 45-60. (doi:10.1542/peds.109.1.45)

11 Suwa S \& Tachibana K. Standard growth charts for height and weight of Japanese children from birth to 17 years based on a cross-sectional survey of national data. Clinical Pediatric Endocrinology 19932 87-97. (doi:10.1297/cpe.2.87)

12 Greulich W \& Pyle S. Radiographic atlas of skeletal development of the hand and wrist. Palo Alto, CA: Stanford University, 1959.

13 Wit JM, Rekers-Mombarg LT, Cutler GB, Crowe B, Beck TJ, Roberts K, Gill A, Chaussain JL, Frisch H, Yturriaga R et al. Growth hormone (GH) treatment to final height in children with idiopathic short stature: evidence for a dose effect. Journal of Pediatrics 2005146 45-53. (doi:10.1016/j.jpeds.2004.08.055)

14 Alatzoglou KS, Turton JP, Kelberman D, Clayton PE, Mehta A, Buchanan C, Alwyn S, Crowne EC, Christensen HT, Hertel NT et al. Expanding the spectrum of mutations in GH1 and GHRHR: genetic screening in a large cohort of patients and congenital isolated growth hormone deficiency. Journal of Clinical Endocrinology and Metabolism 200994 3191-3199. (doi:10.1210/jc.2008-2783)

15 Giavoli C, Porretti S, Ferrante E, Cappiello V, Ronchi CL, Travaglini P, Epaminonda P, Arosio M \& Beck-Peccoz P. Recombinant hGH replacement therapy and the hypothalamus-pituitary-thyroid axis in children with GH deficiency: when should we be concerned about the occurrence of central hypothyroidism? Clinical Endocrinology 200359 806-810. (doi:10.1046/j.1365-2265.2003.01892.x)

16 Agha A, Walker D, Perry L, Drake WM, Chew SL, Jenkins PJ, Grossman AB \& Monson JP. Unmasking of central hypothyroidism following growth hormone replacement in adult hypopituitary patients. Clinical Endocrinology 200766 72-77. (doi:10.1111/j.13652265.2006.02688.x)

17 Behan LA, Monson JP \& Agha A. The interaction between growth hormone and the thyroid axis in hypopituitary patients. Clinical Endocrinology 201174 281-288. (doi:10.1111/j.1365-2265.2010.03815.x)

18 Smyczynka J, Hilczer M, Stawerska R \& Lewinski A. Thyroid function in children with growth hormone $(\mathrm{GH})$ deficiency during the initial phase of GH replacement therapy - clinical implications. Thyroid Research 20103 2. (doi:10.1186/1756-6614-3-2)

19 Walvoord EC, Rosenman MB \& Eugster EA. Prevalence of adrenocorticotropin deficiency in children with idiopathic growth hormone deficiency. Journal of Clinical Endocrinology and Metabolism 200489 5030-5034. (doi:10.1210/jc.2004-0364)

20 Giavoli C, Bergamaschi S, Ferrante E, Ronchi CL, Lania AG, Rusconi R, Spada A \& Beck-Peccoz P. Effect of growth hormone deficiency and recombinant hGH (rhGH) replacement on the hypothalamic-pituitary-adrenal axis in children with idiopathic isolated GH deficiency. Clinical Endocrinology 200868 247-251. (doi:10.1111/j.1365-2265. 2007.03029.x)

21 Donadieu J, Rolon M-A, Pion I, Thomas C, Doz F, Barkaoui M, Robert A Deville A, Mazingue F, David M et al. Incidence of growth hormone deficiency in pediatric-onset Langerhans cell histiocytosis: efficacy and safety of growth hormone treatment. Journal of Clinical Endocrinology and Metabolism 200489 604-609. (doi:10.1210/jc.2003-030907)

22 Carpinteri R, Patelli I, Casaneuva FF \& Giustina A. Inflammatory and granulomatous expansive lesions of the pituitary. Best Practice \& Research. Clinical Endocrinology \& Metabolism 200923 639-650. (doi:10.1016/j.beem.2009.05.009)

23 Coya R, Vela A, Pérez de Nanclares G, Rica I, Castaño L, Busturia MA, Martul P \& GEDPIT Group Panhypopituitarism: genetic versus acquired etiological factors. Journal of Pediatric Endocrinology and Metabolism 2007 20 27-36. (doi:10.1515/JPEM.2007.20.1.27)

24 Hanew K, Tachibana K, Yokoya S, Fujieda K, Tanaka T, Igarashi Y, Shimatsu A, Tanaka H, Tanizawa T, Teramoto A et al. Clinical characteristics, etiologies and pathophysiology of patients with severe short stature with severe GH deficiency: questionnaire study on the data registered with the foundation for growth science, Japan. Endocrine Journal 200653 259-265. (doi:10.1507/endocrj.53.259)

25 Bierich JR. Aetiology and pathogenesis of growth hormone deficiency. Bailliere's Clinical Endocrinology and Metabolism 19926 491-511. (doi:10.1016/S0950-351X(05)80108-9)

26 Ochi M, Morikawa M, Yoshimoto M, Kinoshita E \& Hayashi K. Growth retardation due to idiopathic growth hormone deficiencies: MR findings in 24 patients. Pediatric Radiology 199222 477-480. (doi:10.1007/BF02012987)

Received 5 August 2013

Revised version received 22 September 2013

Accepted 2 October 2013 